\title{
Satisfacción de los estudiantes de epidemiología de medicina con el proceso docente
}

Carmen Amezcua-Prieto, Eladio Jiménez-Mejías, Virgnia Martínez-Ruiz, Rocío Olmedo-Requena, José Juan Jiménez-Moleón, Pablo Lardelli-Claret
Introducción. La implantación del proceso de Bolonia ha supuesto un cambio metodológico en la universidad española en los últimos tiempos. Ello revierte en la calidad de la docencia, específicamente en la titulación de medicina, que se ha ido transformando desde una metodología docente tradicional y unidireccional a otra, más actual, donde el estudiante ocupa un papel principal en el proceso de aprendizaje y donde la evaluación continuada va adquiriendo cada vez más protagonismo.

Sujetos y métodos. En el curso académico 2010-2011 se evaluó, mediante el 'cuestionario ad hoc de mejora de la docencia', el grado de satisfacción y participación del alumnado en la asignatura de 'Epidemiología y demografía sanitaria', materia obligatoria de tercer curso de medicina en la Universidad de Granada, y se relacionó con el rendimiento académico obtenido. Un total de 215 alumnos contestaron al cuestionario. La asociación entre la implicación del alumnado y su satisfacción con este modelo de evaluación y su rendimiento académico se evaluó mediante modelos de regresión lineal múltiple.

Resultados. El 40,8\% de los estudiantes indicó un 'muy elevado grado de satisfacción' con el sistema de evaluación continua. Se observó que, a mayor asistencia a clases de teoría, mayor fue la calificación obtenida en el examen final $(p<0,05$ y $p<0,001$, respectivamente). La implicación del alumnado en las actividades docentes programadas se asoció positivamente con la calificación obtenida en el examen final $(p<0,001)$.

Conclusión. La inclusión de un sistema de evaluación basado en la evaluación continuada favoreció un mayor rendimiento académico del alumnado.

Palabras clave. Evaluación continua. Medicina. Nivel de asistencia. Nivel de participación. Nivel de satisfacción. Rendimiento académico.

\section{Medicine epidemiology students satisfaction with the teaching process}

Introduction. The implementation of the Bolonia's process has meant a methodological change in the Spanish university during the last years. This has affected the teaching quality, specifically in the degree of Medicine, which has changed from a traditional and unidirectional teaching methodology into another situation, in which the students acquire importance in the learning process and the continuous evaluation gets a more important role.

Subjects and methods. In the academic year 2010-2011 the student level of satisfaction and participation in the subject 'Epidemiology and Health Demography' (an obligatory study course from the third year of the degree) and their relationship with the academic performance achieved were assessed by the 'Ad hoc questionnaire of teaching improvement'. A total of 215 students answered the questionnaire. The association between the students' implication and their level of satisfaction with this evaluation system and their academic performance were evaluated with multiple lineal regression models.

Results. The $40.8 \%$ of students indicated a very high level of satisfaction with the continuous evaluation system. It was observed that the higher attendance to lectures the higher marks obtained at the final test $(p<0.05$ and $p<0.001$, respectively). Also, the students implication in the activities scheduled were positively associated with their qualifications at the final test $(p<0.001)$.

Conclusion. The use of a continuous evaluation system in 'Epidemiology and Health Demography' resulted in a better academic performance by the students.

Key words. Academic performance. Continuous evaluation. Level of attendance. Level of participation. Level of satisfaction. Medicine.
Departamento de Medicina Preventiva y Salud Pública. Facultad de Medicina. Universidad de Granada. Granada, España.

Correspondencia: Dra. Carmen Amezcua Prieto. Departamento de Medicina Preventiva y Salud Pública. Facultad de Medicina. Universidad de Granada. Avda. Madrid, s/n. E-18112 Granada.

E-mail:

carmezcua@ugr.es

Agradecimientos:

A los alumnos de la Facultad de Medicina de la Universidad de Granada que cursaron la asignatura 'Epidemiología y demografía sanitaria' en 2010-2011, y que voluntariamente cumplimentaron el cuestionario necesario para realizar esta investigación.

Conflicto de intereses: No declarado.

Competing interests: None declared.

(c) 2015 FEM 


\section{Introducción}

La declaración de la conferencia de ministros participantes en el proceso de Bolonia, en 1999, supuso un acuerdo donde se establecieron las bases para construir en Europa un espacio común para la educación superior. En ella, el alumno se concibe como eje principal del sistema educativo, que aprende por descubrimiento y se fortalece con el trabajo desempeñado por el estudiante tanto individualmente como en grupo. En definitiva, el propósito final es mejorar la calidad de la enseñanza y la competitividad en el ámbito internacional [1]. Pese a ello, al sistema universitario español le acechan dos críticas principales: una creciente, pero aún insuficiente, atención por parte del profesorado al proceso de enseñanza-aprendizaje en sí mismo y una metodología docente tradicional basada predominantemente en clases magistrales o unidireccionales [2]. Algunos autores han hecho propuestas de cambio del modelo tradicional de enseñanza-aprendizaje en el Grado de Medicina, al objeto de conseguir una mayor implicación tanto del profesorado como, sobre todo, del alumnado en su propio proceso educativo y mejorar así la calidad de la docencia $[3,4]$. Para ello, una de las posibles estrategias propuestas desde algunos ámbitos de la pedagogía es la incorporación de nuevos sistemas de evaluación, por ejemplo, los denominados sistemas de evaluación continuada [5-7], hecho éste sobre el que ya se está trabajando desde la implantación de los grados en la universidad española, pues las asignaturas deben contar con un conjunto de 'actividades y pruebas de evaluación continua', según acuerdo de gobierno de 23 de octubre de 2008 [8].

La evaluación continua proporciona la oportunidad de consolidar conocimientos y desarrollar y poner en práctica habilidades relacionadas con los resultados de aprendizaje, además de proporcionar información sobre el proceso de aprendizaje y reconocer el trabajo del alumno, contribuyendo a dotar de más elementos al profesorado a la hora de calificar el papel de los alumnos en la asignatura [8].

La epidemiología y la demografía sanitaria son herramientas de la salud pública incluidas en el currículo de cualquier titulación del ámbito de ciencias de la salud [9-13]. Se imparte como materia troncal en las 28 facultades de medicina del territorio nacional, y así se establece en las directrices generales propias del título de Licenciado en Medicina [14]. Dicha asignatura ha formado parte del tercer curso de la licenciatura de medicina en la Universidad de Granada, según se contempla en el plan de estudios de 19 de noviembre de 2001 de Licen- ciado en Medicina [15] y en los próximos años pasará a ser un módulo de la asignatura de 'Medicina preventiva y salud pública' en quinto curso [9].

Por las especiales características de una materia como la epidemiología y la demografía sanitaria, en la que es necesario la adquisición acumulativa de conceptos para poder resolver problemas más complejos [16], nos pareció pertinente emplear como instrumento de evaluación un sistema de evaluación continuada en el que se valoraban los siguientes aspectos: asistencia a clase, puntos positivos a respuestas de preguntas planteadas por el profesor, participación en seminarios y elaboración de preguntas de respuesta múltiple. Con ello se incentivaba y reconocía el trabajo del alumno a lo largo de todo el curso académico y su evaluación no quedaba supeditada en exclusiva a la calificación obtenida en el examen final.

Los trabajos realizados sobre investigación educativa en la universidad española han sido llevados a cabo en disciplinas tales como pedagogía y psicología, no tanto en el área de ciencias de la salud y, en especial, en la titulación de medicina [17]. En 1996 se estudió, en la Facultad de Medicina de la Universitat Autònoma de Barcelona, la asociación existente entre la asistencia a clase y el rendimiento académico para distintas asignaturas de la titulación [18]. Por otro lado, la metodología docente de la asignatura de epidemiología ha sido evaluada en la licenciatura de farmacia, en el curso académico 2012-2013, en la Universidad de Alcalá, en Guadalajara [19]. Sin embargo, no hemos encontrado estudios en nuestro país que hayan valorado de forma conjunta la asociación entre la asistencia y participación en clase por parte del alumnado y, por otro lado, su satisfacción con la materia y el rendimiento académico finalmente obtenido.

El objetivo de este trabajo fue conocer el nivel de satisfacción del alumnado con la metodología docente empleada en 'Epidemiología general y demografía sanitaria’ y valorar la asociación entre el empleo de un sistema de evaluación continuada con el rendimiento académico obtenido por el alumnado.

\section{Sujetos y métodos}

En el curso académico 2010-2011, 330 alumnos se matricularon de la asignatura troncal 'Epidemiología general y demografía sanitaria' en la Facultad de Medicina de la Universidad de Granada, compuesta de seis créditos (cuatro teóricos y dos prácticos).

La materia constaba de 40 temas de teoría impartidos en clases de 55 minutos de duración y 10 
sesiones de prácticas de dos horas cada una. Había dos grupos de aproximadamente 150 alumnos para recibir la docencia teórica y 10 grupos de 30 alumnos para las sesiones prácticas.

La metodología docente empleada en la asignatura de epidemiología incluía las siguientes actividades, todas ellas puntuadas, para realizar una evaluación continua:

- Clases de teoría: evaluación positiva (de 1 a 5 puntos) por respuestas a preguntas elaboradas por el profesor en clase.

- Clases prácticas: evaluación positiva por asistencia a clase (0,5 puntos por asistencia).

- Seminarios: evaluados positivamente de 1 a 10 puntos, según presentación del alumno.

- Banco de preguntas: de donde se extraía un 5\% de las preguntas del examen final de junio; evaluación positiva de 0,5 puntos por pregunta bien formulada.

- Examen de prácticas: 40 preguntas de respuesta múltiple y cinco alternativas de respuesta; evaluación positiva de 1 punto por cada respuesta acertada y evaluación negativa de 1 punto por cada cuatro preguntas incorrectas.

- Examen de teoría: 70 preguntas de respuesta múltiple y misma evaluación que para el examen práctico.

La nota final de la asignatura se obtuvo aplicando la siguiente fórmula: suma de puntos de evaluación positiva. Se consideró la asignatura como suspendida con menos de 5 puntos; aprobado, de 5 a 6,9 puntos; notable, de 7 a 8,9 puntos, y sobresaliente, con 9 o más puntos.

El cuestionario de mejora de la docencia (CMD), elaborado específicamente para conocer el grado de satisfacción y participación en la asignatura de 'Epidemiología general y demografía sanitaria' del curso académico 2010-2011, fue un cuestionario $a d$ hoc constituido por 11 preguntas con cinco alternativas de respuesta (Tabla I). De ellas, cinco preguntas se refirieron a la satisfacción del alumnado (con la organización del curso, con las clases teóricas, con los textos docentes y las presentaciones expuestas en clase, con la evaluación general, y grado de utilidad de las actividades realizadas en clase), todas ellas con cinco alternativas de respuesta (muy bajo, bajo, normal, elevado y muy elevado). Cuatro preguntas se relacionaron con la participación del alumnado: asistencia a clases teóricas $(<6-10,11-15$, $16-20$ y > 20 días), consulta de la guía docente (nunca, una vez al inicio del curso, una vez al final del curso, más de una vez a lo largo del curso, más de una vez al final del curso), tiempo de dedicación a
Tabla I. Cuestionario de mejora de la docencia (CMD), curso 2010-2011.

1. Indica tu grado de satisfacción con la información suministrada sobre la organización del curso:

A) Muy bajo; B) Bajo; C) Regular; D) Alto; E) Muy alto

2. Indica tu grado de satisfacción con la técnica docente aplicada en las clases teóricas:

A) Muy bajo; B) Bajo; C) Regular; D) Alto; E) Muy alto

3. Indica el grado de utilidad del material docente suministrado en el tablón de docencia:

Sobre la percepción/ satisfacción del alumnado

- Texto: A) Muy poco útil; B) Poco útil; C) Normal; D) Útil; E) Muy útil

- Presentaciones: A) Muy poco útil; B) Poco útil; C) Normal; D) Útil; E) Muy útil

4. Indica tu grado de satisfacción global con el sistema de evaluación aplicado: A) Muy bajo; B) Bajo; C) Regular; D) Alto; E) Muy alto

5. Indica el grado de utilidad con las siguientes actividades realizadas durante el curso:

- Preguntas en clases teóricas: A) Muy útil; B) Útil; C) Normal; D) Poco útil; E) Muy poco útil

- Banco de preguntas: A) Muy útil; B) Útil; C) Normal; D) Poco útil; E) Muy poco útil

- Seminarios: A) Muy útil; B) Útil; C) Normal; D) Poco útil; E) Muy poco útil

6. Indica a cuántas clases teóricas has asistido durante el curso: A) Menos de 5; B) De 6 a 10; C) De 11 a 15; D) De 16 a 20; E) Más de 20

7. Marca aquellas actividades en las que has participado: A) Banco de preguntas; B) Seminarios; C) Preguntas de clase; D) Asistencia a prácticas

Sobre la asistencia/ participación del alumnado

8. ¿Cuántas veces has consultado la guía docente de la asignatura?: A) Nunca; B) Una vez, al inicio del curso; C) Una vez, al final del curso; D) Más de una vez, a lo largo del curso; E) Más de una vez, al final del curso

9. ¿Cómo estimas que ha sido el tiempo que has dedicado (esfuerzo) a esta asignatura?:

A) Muy bajo; B) Bajo; C) Regular; D) Alto; E) Muy alto

10. Indica el grado de dificultad sobre las preguntas planteadas en los exámenes: A) Dificultad excesiva; B) Dificultad moderada; C) Adecuadas; D) Moderadamente fáciles; E) Excesivamente fáciles

Sobre el grado de dificultad en los exámenes y acceso al profesorado

11. Indica el grado de accesibilidad al profesorado que has percibido: A) Muy bajo; B) Bajo; C) Normal; D) Alto; E) Muy alto

12. Propuesta de mejora en la técnica docente

Sobre la introducción de mejoras

13. Propuesta de mejora en material suministrado

14. Propuesta de mejora en el sistema de evaluación

la asignatura (muy bajo, bajo, regular, alto, muy alto) y grado de participación en actividades docentes. El cuestionario también incluyó dos preguntas sobre el grado de dificultad en los exámenes y acceso al profesorado $y$, por último, tres preguntas que recogían la propuesta del alumnado sobre las mejoras que podrían introducirse en la técnica docente, 
el material suministrado y la evaluación existente de las actividades docentes programadas.

A los alumnos que se presentaron al examen final de junio se les repartió el CMD. Datos como la edad y el sexo no se recogieron en el cuestionario, pero se les solicitaba su identificación voluntaria con el documento nacional de identidad para relacionar su nivel de satisfacción y grado de participación con la calificación obtenida en el examen final.

Para el análisis de los datos, se calculó la media y desviación estándar de variables cuantitativas continuas, y para las variables cualitativas, la frecuencia y distribución, así como sus intervalos de confianza al 95\%. Posteriormente, se realizó un análisis de regresión lineal considerando siempre como variable dependiente la nota final del examen oficial (que no incorporaba en su cómputo la nota del resto de actividades programadas para la asignatura). Para el análisis de los datos se utilizó el programa estadístico Stata v.12.

\section{Resultados}

$\mathrm{Al}$ examen final de junio se presentaron 266 alumnos, 215 (80,8\%) cumplimentaron el CMD tras finalizar el examen. La distribución por sexos fue: $65,1 \%$ mujeres y $34,8 \%$ hombres.

En la tabla II aparecen los resultados recogidos del CMD sobre la satisfacción, participación, percepción del grado de dificultad e incorporación de mejoras metodológicas del alumnado, que se resumen a continuación:

- Percepción/satisfacción del alumnado. El 65,0\% de los estudiantes consideró muy útil el material docente suministrado (en el tablón de docencia, plataforma de uso académico para la Universidad de Granada) y el $41,8 \%$ y el $40,8 \%$, respectivamente, indicaron un elevado y muy elevado grado de satisfacción con el sistema de evaluación continua. De entre las actividades programadas en la asignatura, las de mayor utilidad percibida por los alumnos $(43,9 \%)$ fueron las preguntas elaboradas por el profesor en clases teóricas.

- Asistencia/participación del alumnado. El 46,2\% del alumnado acudió a más de 20 clases teóricas (de un total de 40). Sólo un 10,5\% participó en todas las actividades organizadas en la asignatura (clases de teoría y prácticas, respuesta a preguntas de clase, elaboración propia de preguntas para el banco de preguntas y preparación de seminarios). Además, el 66\% del alumnado consultó la guía docente de la asignatura más de una vez a lo largo del curso académico y el $42,3 \%$ consi- deró que el tiempo de dedicación a la asignatura fue elevado.

- Grado de dificultad en los exámenes y acceso al profesorado. El 67,0\% del alumnado consideró que los exámenes de respuesta múltiple fueron de dificultad moderada. Por otra parte, el 47,9\% consideró el acceso al profesorado como alto, y el 30,7\%, como muy alto.

- Introducción de mejoras en la asignatura desde el punto de vista del alumnado. Siete de cada diez alumnos que contestaron al CMD estuvieron satisfechos con la técnica docente empleada. Seis de cada diez alumnos se mostraron satisfechos con el material docente y el sistema de evaluación. Las propuestas que los alumnos manifestaron para mejorar la asignatura fueron: una mayor explicación teórica y más ejemplos prácticos.

\section{Calificaciones}

Con respecto a la calificación obtenida en el examen teórico (sin considerar la puntuación del resto de actividades), la nota media fue de 39,64 $\pm 0,88$ (rango: 5-68 puntos), de un total de 70 puntos. La evaluación continua, que consideraba todas las actividades docentes programadas para la asignatura, mostró que el $81,9 \%$ de los alumnos superó la asignatura en la convocatoria de julio: $7,9 \%(n=17)$ con matrícula de honor, 9,8\% $(n=21)$ con sobresaliente, $26,5 \%(n=57)$ con notable y $35,8 \%(n=77)$ con aprobado.

La relación entre la satisfacción y participación del alumnado con el rendimiento académico puede apreciarse en la tabla III. En el análisis bivariante se observó que, a mayor asistencia a clases de teoría (16-20 veces o $>20$ veces), mayor fue también la calificación obtenida en el examen final $(p<0,05$ y $p<$ 0,001 , respectivamente). En este mismo análisis, se observó un gradiente dosis-respuesta entre el nivel de satisfacción con la organización del curso y la calificación obtenida en el examen. Además, la participación en todas las actividades docentes programadas en la asignatura se asoció positivamente con una mayor puntuación en el examen de contenidos teóricos final $(p<0,001)$.

Teniendo en cuenta el efecto de la asistencia a clase, la satisfacción con la organización del curso y el grado de participación en las actividades docentes programadas (análisis de regresión lineal múltiple), como puede verse en la tabla III, únicamente la asistencia a clase y la participación en todas las actividades docentes programadas en la asignatura se asociaron de forma positiva y estadísticamente significativa con una mayor calificación obtenida 
Tabla II. Descripción de las variables de satisfacción y participación del alumnado

\begin{tabular}{|c|c|c|c|c|c|c|}
\hline \multirow{11}{*}{$\begin{array}{l}\text { Grado de } \\
\text { satisfacción }\end{array}$} & & $\begin{array}{c}1 \\
\text { (muy bajo, } \\
\text { muy poco útil } \\
\text { o muy fácil) }\end{array}$ & $\begin{array}{c}2 \\
\text { (bajo, } \\
\text { poco útil } \\
\text { o algo fácil) }\end{array}$ & $\begin{array}{c}3 \\
\text { (normal) }\end{array}$ & $\begin{array}{l}4 \\
\text { (alto, } \\
\text { útil o } \\
\text { difícil) }\end{array}$ & $\begin{array}{c}5 \\
\text { (muy alto, } \\
\text { muy útil o } \\
\text { muy difícil) }\end{array}$ \\
\hline & Organización $(n=201)$ & 0 & $6(3,0 \%)$ & $15(7,5 \%)$ & $97(48,3 \%)$ & $83(41,3 \%)$ \\
\hline & Clases teóricas $(n=199)$ & $7(3,6 \%)$ & $3(1,5 \%)$ & $35(17,8 \%)$ & $110(55,9 \%)$ & $42(21,3 \%)$ \\
\hline & Textos en clase $(n=200)$ & 0 & $4(2,0 \%)$ & $19(9,5 \%)$ & $46(23,0 \%)$ & $131(65,0 \%)$ \\
\hline & Presentaciones $(n=195)$ & $3(1,5 \%)$ & $8(4,1 \%)$ & $69(35,4 \%)$ & $63(32,3 \%)$ & $52(26.7 \%)$ \\
\hline & Preguntas de clase $(n=198)$ & $11(5,6 \%)$ & $7(3,5 \%)$ & $59(29,8 \%)$ & $87(43,9 \%)$ & $34(17,2 \%)$ \\
\hline & Banco de preguntas $(n=199)$ & $10(5,0 \%)$ & $17(8,5 \%)$ & $55(27,6 \%)$ & $74(37,2 \%)$ & $43(21,6 \%)$ \\
\hline & Seminarios $(n=197)$ & $7(3,5 \%)$ & $19(9,6 \%)$ & $59(30,0 \%)$ & $62(31,5 \%)$ & $50(25,4 \%)$ \\
\hline & Dificultad de exámenes tipo test $(n=197)$ & 0 & 0 & $41(20,8 \%)$ & $132(67,0 \%)$ & $24(12,8 \%)$ \\
\hline & Sistema de evaluación $(n=201)$ & 0 & $5(2,5 \%)$ & $30(14,9 \%)$ & $84(41,8 \%)$ & $82(40,8 \%)$ \\
\hline & Accesibilidad al profesorado $(n=192)$ & $2(1,0 \%)$ & 0 & $39(20,3 \%)$ & $92(47,9 \%)$ & $59(30,7 \%)$ \\
\hline \multirow{5}{*}{$\begin{array}{l}\text { Grado de } \\
\text { participación }\end{array}$} & Asistencia a clases teóricas ( $n=199)$ & $\begin{array}{l}\text { Menos de 6: } \\
29(14,6 \%)\end{array}$ & $\begin{array}{l}\text { De } 6 \text { a } 10: \\
26(13,1 \%)\end{array}$ & $\begin{array}{l}\text { De } 11 \text { a } 15: \\
26(13,1 \%)\end{array}$ & $\begin{array}{l}\text { De } 16 \text { a } 20: \\
26(13,1 \%)\end{array}$ & $\begin{array}{l}\text { Más de 20: } \\
92(46,2 \%)\end{array}$ \\
\hline & Actividades $(n=199)$ & & & & & \\
\hline & $\begin{array}{l}\text { 1. Banco de preguntas } \\
\text { 2. Seminarios } \\
\text { 3. Preguntas de clase } \\
\text { 4. Asistencia a prácticas }\end{array}$ & $\begin{array}{l}1 \text { actividad: } \\
67(33,7 \%)\end{array}$ & $\begin{array}{l}2 \text { actividades: } \\
90(42,5 \%)\end{array}$ & $\begin{array}{l}3 \text { actividades: } \\
21(10,5 \%)\end{array}$ & $\begin{array}{l}4 \text { actividades: } \\
21(10,5 \%)\end{array}$ & \\
\hline & Consulta de la guía ( $n=197$ ) & $\begin{array}{l}\text { Nunca: } \\
7(3,5 \%)\end{array}$ & $\begin{array}{l}\text { Una vez, } \\
\text { al inicio } \\
\text { del curso: } \\
29(14,7 \%)\end{array}$ & $\begin{array}{l}\text { Una vez, } \\
\text { al final } \\
\text { del curso: } \\
19(9,6 \%)\end{array}$ & $\begin{array}{l}\text { Más de una } \\
\text { vez, a lo largo } \\
\text { del curso: } \\
130(66,0 \%)\end{array}$ & $\begin{array}{l}\text { Más de una } \\
\text { vez, al final } \\
\text { del curso: } \\
12(6,1 \%)\end{array}$ \\
\hline & $\begin{array}{l}\text { Tiempo de dedicación } \\
\text { a la asignatura }(n=194)\end{array}$ & $\begin{array}{l}\text { Muy bajo: } \\
1(0,5 \%)\end{array}$ & $\begin{array}{c}\text { Bajo: } \\
7(3,6 \%)\end{array}$ & $\begin{array}{c}\text { Normal: } \\
102(52,6 \%)\end{array}$ & $\begin{array}{c}\text { Alto: } \\
82(42,3 \%)\end{array}$ & $\begin{array}{l}\text { Muy alto: } \\
2(1 \%)\end{array}$ \\
\hline
\end{tabular}

en el examen $(p<0,001$ y $p<0,05$, respectivamente). En este último análisis, sin embargo, el grado de satisfacción, aun asociándose positivamente con el resultado en el examen, no fue estadísticamente significativo.

\section{Discusión}

El 80,8\% de los alumnos que se presentaron al examen final de junio de la asignatura 'Epidemiología general y demografía sanitaria' en el curso académico 2010-2011 contestaron al CMD. A partir de ello, se pudo describir el grado de satisfacción, participación, dificultad y acceso al profesorado. Sin em- bargo, para analizar la relación entre la satisfacción y la participación del alumnado con el rendimiento académico (calificación obtenida en el examen final), se usó el total de alumnos identificados con su documento nacional de identidad $(n=199)$, información del 74,8\% de los presentados. En consonancia con lo referido por otras fuentes en cuanto a la distribución por sexos en las titulaciones relacionadas con las ciencias de la salud [19,20], en torno al $66 \%$ de nuestra muestra se componía por mujeres. La información sobre la variable sexo la obtuvimos gracias al cruce de la base de datos obtenida del CMD con la confeccionada durante el curso académico, donde se notificaba la asistencia a clase y la puntuación obtenida en la evaluación continua. 
Tabla III. Relación entre el grado de satisfacción y participación con el rendimiento académico alcanzado $(n=199)$.

\begin{tabular}{|c|c|c|c|c|c|}
\hline & \multicolumn{3}{|c|}{ Resultado académico en el examen final (oficial) } & \multirow{2}{*}{$\begin{array}{l}\text { Coeficiente } \\
\text { ajustado }^{\text {a }}\end{array}$} & \multirow{2}{*}{$\begin{array}{c}\text { IC } 95 \% \\
\text { ajustado a }\end{array}$} \\
\hline & Categorías & $\begin{array}{l}\text { Coeficiente } \\
\text { crudo }\end{array}$ & $\begin{array}{l}\text { IC } 95 \% \\
\text { crudo }\end{array}$ & & \\
\hline \multirow{5}{*}{$\begin{array}{l}\text { Asistencia } \\
\text { a clase }\end{array}$} & $<6$ & 0 & Referencia & 0 & Referencia \\
\hline & $6-10$ & $-0,41$ & $-7,34-6,52$ & $-0,55$ & $-7,86-6,77$ \\
\hline & $11-15$ & 4,69 & $-2,03-11,41$ & 3,98 & $-3,00-10,97$ \\
\hline & $16-20$ & 9,88 & $3,09-16,66^{b}$ & 9,45 & $2,22-16,70^{b}$ \\
\hline & $>20$ & 13,37 & $7,85-18,90^{c}$ & 11,56 & $5,51-17,62^{c}$ \\
\hline \multirow{4}{*}{$\begin{array}{l}\text { Satisfacción por } \\
\text { la organización }\end{array}$} & Muy baja/baja & 0 & Referencia & 0 & Referencia \\
\hline & Normal & 15,30 & $-3,31-34,21$ & 8,36 & $-8,93-25,66$ \\
\hline & Alta & 18,90 & $1,43-36,35^{b}$ & 11,46 & $-4,47-27,39$ \\
\hline & Muy alta & 21,22 & $3,72-38,72^{b}$ & 10,74 & $-5,37-26,86$ \\
\hline \multirow{4}{*}{$\begin{array}{l}\text { Grado de } \\
\text { participación } \\
\text { en actividades }\end{array}$} & 0 & 0 & Referencia & 0 & Referencia \\
\hline & 1 & 3,85 & $-0,34-8,06$ & 1,03 & $-3,13-5,20$ \\
\hline & 2 & 5,76 & $-0,33-11,85$ & 1,11 & $-4,87-7,09$ \\
\hline & 3 & 14,05 & $7,74-20,37^{c}$ & 9,06 & $2,63-15,49^{b}$ \\
\hline \multirow{4}{*}{$\begin{array}{l}\text { Tiempo de } \\
\text { dedicación }\end{array}$} & Muy bajo/bajo & 0 & Referencia & 0 & Referencia \\
\hline & Normal & 7,50 & $-2,24-17,25$ & - & - \\
\hline & Alto & 7,08 & $-2,79-16,95$ & - & - \\
\hline & Muy alto & 6,36 & $-13,56-26,28$ & - & - \\
\hline
\end{tabular}

IC 95\%: intervalo de confianza al 95\%. ${ }^{a}$ Modelo de regresión lineal múltiple ajustado por asistencia a clase, satisfacción con la organización y participación en las actividades programadas para el curso académico; ${ }^{b} p<0,05$; ${ }^{c} p<0,001$. ron acudir a clase ( $>16$ veces de un total de 40$)$ y participar en todas las actividades docentes programadas para el curso académico (preguntas de clase, asistencia a prácticas, seminarios y bancos de preguntas). Otros estudios llevados a cabo en el ámbito universitario también destacan que la asistencia a clase [18,22-24] y la participación del alumnado [25] influyen positivamente en la calificación obtenida por el alumno.

El nivel de participación en las tareas diseñadas para la asignatura refleja el grado de motivación del alumnado, que a su vez repercute en el resultado final obtenido, al adquirir un mayor compromiso e implicación personal con el aprendizaje [26-28]. Debemos destacar que el $40,8 \%$ de la muestra de estudio acudió a menos de 15 clases teóricas, lo que correspondía con una falta de asistencia a clase del $37,5 \%$. Algunos autores reflejan que el absentismo a clase en la universidad está muy relacionado con el bajo rendimiento académico [23,29]. En contraposición, el $46,2 \%$ acudió a más de la mitad de las clases teóricas. Investigadores de la Universidad de Granada reflejan que los alumnos que asisten a clase son estudiantes comprometidos, persistentes y con autonomía e independencia para aprender [30].

La relación entre las variables independientes del estudio (asistencia a clase, satisfacción con la organización, grado de participación en las actividades, tiempo de dedicación) y la variable dependiente 'rendimiento académico' requiere una breve reflexión. Dichas asociaciones se han abordado en esta investigación utilizando como variable de resultado la calificación obtenida en el examen final, cuya puntuación máxima fue de 70 puntos.

El CMD elaborado ad hoc no se ha validado previamente. Se construyó con el objetivo de conocer la opinión del alumnado con la organización de la asignatura y con el sistema de evaluación empleado, como antes se ha realizado en otros estudios en el ámbito universitario [29,31]. Además, somos conscientes del más que probable sesgo tanto de selección como de información que subyace al emplear el citado cuestionario (de cumplimentación voluntaria y no totalmente anónima) al final del examen final. El primer sesgo provoca que los resultados obtenidos únicamente sean extrapolables, y con la debida cautela, a los alumnos matriculados en este curso académico. Respecto al sesgo de información, la concordancia de nuestros resultados con los de estudios previos $[4,18]$ y el hecho de haber incluido en el modelo multivariante las posibles variables que $a$ priori pueden comportarse como potenciales confusores de la calificación final, son argumentos a favor de la validez de los resultados. 
En definitiva, la asistencia a clase y la participación en las actividades programadas en la asignatura de 'Epidemiología general y demografía sanitaria', de tercer curso de la licenciatura de medicina, fueron las mejores fuentes de predicción de un mayor rendimiento académico. En el proceso de enseñanzaaprendizaje, la inclusión de un sistema de evaluación basado en la 'evaluación continua' contribuyó a un mayor rendimiento académico del alumnado.

Bibliografía

1. Documento-marco. La integración del sistema universitario español en el espacio europeo de enseñanza superior. Madrid: Ministerio de Educación, Cultura y Deporte; 2003. URL: http://www.eees.es/pdf/Documento-Marco_10_Febrero.pdf.

2. González I. Aproximación a una formación académica de calidad: el punto de vista de los estudiantes universitarios. Revista Electrónica de Investigación Psicoeducativa y Psicopedagógica 2003; 1: 1-22.

3. Palés JL, Rodríguez de Castro F. Retos de la formación médica de grado. Educ Med 2006; 9: 159-72.

4. Gal-Iglesias B, Busturia-Berrade I, Garrido-Astray MC. Nuevas metodologías docentes aplicadas al estudio de la fisiología y la anatomía: estudio comparativo con el método tradicional. Educ Med 2009; 12: 117-24.

5. Delgado AM, Oliver R. La evaluación continua en un nuevo escenario docente. Revista de Universidad y Sociedad del Conocimiento 2006. URL: http://www.uoc.edu/rusc/3/1/dt/ esp/delgado_oliver.pdf.

6. Manso-Martínez J. Reflexiones sobre un nuevo modelo de profesor universitario en medicina. Una visión crítica de la enseñanza médica. URL: http://www.fac.org.ar/scvc/llave/ edu/manso/mansoe.htm.

7. Calderón-Patier C, Escalera-Izquierdo G. La evaluación de la docencia ante el reto del Espacio Europeo de Educación Superior (EEES). Educación XXI: Revista de la Facultad de Educación 2008; 11: 237-56.

8. Evaluación continua en el EEES. Vicerrectorado de Espacio Europeo y Planificación Docente. Vicerrectorado de Calidad e Innovación Docente. UNED. URL: http://portal.uned.es/ pls/portal/docs/PAGE/UNED_MAIN/LAUNIVERSIDAD/ VICERRECTORADOS/CALIDAD_E_INNOVACION/ INNOVACION_DOCENTE/IUED/DOCUMENTOS/ EVALUACION_CONTINUA_EEES_UNED.PDF.

9. Programa Verifica (1) de Grado en Medicina (ANECA, 2009). URL: http://grados.ugr.es/medicina/pages/infoacademica/ archivos/verificamedicina.

10. Programa Verifica (2) de Grado en Enfermería (ANECA, 2009). URL: http://grados.ugr.es/enfermeria/pages/infoacademica/ verificaenfermeria.

11. Programa Verifica (3) de Grado en Farmacia (ANECA, 2009). URL: http://grados.ugr.es/farmacia/pages/infoacademica/ archivos/verificafarmacia.

12. Programa Verifica (4) de Grado en Fisioterapia (ANECA, 2009). URL: http://grados.ugr.es/fisioterapia/pages/infoacademica/ verificafisioterapia.

13. Programa Verifica (5) de Grado en Terapia Ocupacional.
URL: http://vicengp.ugr.es/pages/_grados-verificados/55ter apiaocupacionalverificado.

14. Real Decreto $1417 / 1990$, de 26 de octubre, por el que se establece el título universitario oficial de Licenciado en Medicina y las directrices generales propias de los planes de estudios conducentes a la obtención de aquél. Boletín Oficial del Estado n. ${ }^{\circ} 278$, de 20 de noviembre de 1990, p. 34343-5.

15. Resolución de 19 de noviembre de 2001, de la Universidad de Granada, por la que se hace público el plan de estudios de Licenciado en Medicina. Boletín Oficial del Estado n. ${ }^{\circ} 300$, de 15 de diciembre de 2001, p. 47806-15.

16. Almeida-Filho $\mathrm{N}$. Por una epidemiología con (más que) números: cómo superar la falsa oposición cuantitativo-cualitativo. Salud Colectiva 2007; 3: 229-33.

17. Benito A, Cruz A. Nuevas claves para la docencia universitaria en el Espacio Europeo de Educación Superior. Madrid: Narcea; 2007.

18. Pérez J, Graell S. Asistencia a clase y rendimiento académico en estudiantes de medicina. La experiencia de la Universidad Autónoma de Barcelona. Educ Med 2004; 7: 85-9.

19. Albert R, Dolado JJ, Bosch JL, Cabrillo F, Chorén P, Felgueroso F, et al. La feminización de las profesiones sanitarias. Bilbao: Fundación BBVA; 2010.

20. Instituto Nacional de Estadística. Estadística de enseñanza universitaria (2012). URL: http://www.ine.es.

21. Tejedor FJ, García-Valcárcel A. Causas del bajo rendimiento del estudiante universitario (en opinión de los profesores y alumnos). Propuestas de mejora en el marco del EEES. Revista de Educación 2007; 342: 443-73.

22. Fernández E. ¿Cuál es el papel del alumnado dentro del proceso de enseñanza-aprendizaje en la universidad? Revista de Educación 2001; 325: 201-7.

23. Cabrera L, Tomás J, Álvarez P, González M. El problema del abandono de los estudios universitarios. Relieve 2006; 12: 171-203.

24. Rodríguez C, Herrera L. Análisis correlacional-predictivo de la influencia de la asistencia a clase en el rendimiento académico universitario. Estudio de caso en una asignatura. Revista de Currículum y Formación del Profesorado 2009; 13: 1-13.

25. García MV, Alvarado JM, Jiménez A. La predicción del rendimiento académico: regresión lineal versus regresión logística. Psicothema 2000; 12: 248-52.

26. González R, Valle A, Rodríguez S, García M, Mendiri P. Programa de intervención para mejorar la gestión de los recursos motivacionales en estudiantes universitarios. Revista Española de Pedagogía 2007; 237: 237-56.

27. Mas C, Medinas M. Motivaciones para el estudio en universitarios. Anales de Psicología 2007; 23: 17-24.

28. Rozendal JS, Minnaert A, Boekaerts M. The influence of teacher perceived administration of self-regulated learning on students' motivation and information processing. Learning and Instruction 2005; 15: 141-60.

29. Álvarez PR, López DP. Un estudio transversal retrospectivo sobre prolongación y abandono de estudios universitarios. Relieve 2006; 12: 105-27.

30. De la Fuente J, Pichardo MC, Justicia F, Berbén A. Enfoques de aprendizaje; autorregulación y rendimiento en tres universidades europeas. Psicothema 2008; 20: 705-11.

31. Rodríguez R, Hernández J, Alonso A, Díez E. El absentismo en la universidad: resultados de una encuesta sobre motivos que señalan los estudiantes para no asistir a clase. Aula Abierta 2003; 82: 117-45. 